\title{
Combinando o método de Pontos Interiores com o método Simplex para a solução de problemas de programação linear
}

\author{
Cecilia Orellana Castro ${ }^{1}$ \\ Universidade Federal do Sul e Sudeste do Pará \\ Manolo Rodriguez Heredia ${ }^{2}$ \\ Universidade Federal do Sul e Sudeste do Pará \\ Aurelio R. L. Oliveira ${ }^{3}$ \\ Universidade Estadual de Campinas
}

\begin{abstract}
Resumo. Quando a matriz $A$ de restrições de um problema de programação linear apresenta posto completo, no método Simplex é usada uma submatriz não singular de $A$ para determinar uma solução básica. Neste contexto, no método de Pontos Interiores, quando são usados métodos iterativos precondicionados, também usa-se uma submatriz não singular de $A$ para calcular o precondicionador Separador. O processo que calcula uma solução básica factível para o método Simplex, a partir de um ponto do método de Pontos Interiores é chamado crossover. Neste trabalho combinam-se ambos os métodos para a solução de problemas de programação linear canalizados, mais precisamente usa-se uma base do precondicionador Separador para determinar se a solução básica associada a ela é factível primal, dual ou infactível no sentido do método Simplex. Resultados numéricos mostram que alguns problemas que não eram resolvidos usando apenas o método de Pontos Interiores, são resolvidos usando a estratégia do crossover. Também observam-se problemas que reduzem consideravelmente o número de iterações do método Simplex quando inicialmente usam o método de Pontos Interiores, isto é, combinam ambos os métodos.
\end{abstract}

Palavras-chave. Método de Pontos Interiores, Método Simplex, crossover

\section{Introdução}

O método Simplex e método de Pontos Interiores (MPI) são reconhecidos como ferramentas eficientes para problemas de programação linear. Uma melhor compreensão do condicionamento das matrizes que surgem no MPI incentivou os pesquisadores a examinar precondicionadores especiais, foi assim como surgiu o precondicionador Separador, [6].

Paralelamente pesquisadores melhoraram o método Simplex, de fato, ambos métodos são eficientes na prática, o método Simplex é mais adequado para problemas densos.

\footnotetext{
${ }^{1}$ ceciliaoc@unifesspa.edu.br.

${ }^{2}$ manolorh@unifesspa.edu.br.

3 aurelio@ime.unicamp.br.
} 
Mesmo que este método faça muitas iterações para convergir, elas são baratas. No entanto, o MPI faz poucas iterações, que geralmente são computacionalmente caras.

No método Simplex existe um conceito muito importante: solução básica, que está associada a uma matriz quadrada não singular chamada base. Neste método, a partir de uma solução básica factível inicial constrói-se uma sequência finita de soluções básicas factíveis de tal maneira que o valor da função objetivo forma uma sequência monótona. No método de Pontos Interiores, quando se trabalha com o precondicionador Separador, também existe o conceito de matriz básica ou base. Embora ambas sejam matrizes não singulares, os conceitos são totalmente diferentes e são explicados na Seção 2.

Neste trabalho, usa-se o conceito de base de ambos os métodos para combiná-los na solução de problemas de programação linear canalizado:

$$
\begin{aligned}
& \text { (P) }: \min c^{T} x, \text { s. a. }\{A x=b, x+s=u, x, s \geq 0\} \\
& \text { (D) }: \max b^{T} y-u^{T} w, \text { s. a. }\left\{A^{T} y-w+z=c, w, z \geq 0, y \in \mathbb{R}^{m}\right\}
\end{aligned}
$$

onde $x, s, w, z \in \mathbb{R}^{n}$ e a matriz $A: m \times n$ que será considerada de posto completo. Mais precisamente, quando os sistemas lineares oriundos do MPI começam a ficar muito mal condicionados apresentando uma convergência lenta, a base do precondicionador Separador no MPI é usada para obter uma base inicial do método Simplex, e desta maneira, atingir a convergência usando este método.

\section{Base no método Simplex e Base do precondicionador Se- parador no MPI primal-dual}

\subsection{Base no método Simplex}

Dado que $A_{m \times n}$ em (1) é de posto completo, existe uma submatriz não singular $\left(A_{B}\right)_{m \times m}$ de $A$, onde $B$ é um conjunto de índices que correspondem às $m$ colunas linearmente independentes chamados índices básicos. Chama-se de índices não básicos associados a um dado vetor $x \in \mathbb{R}^{n}$ os conjuntos $N_{1}=\left\{i: x_{i}=0\right\}$ e $N_{2}=\left\{i: x_{i}=u_{i}\right\}$. Além disso, a matriz $A_{B}$ é chamada de base, no método Simplex. Após uma reordenação das colunas de $A$, podemos considerar que $A=\left[A_{B}, A_{N_{1}}, A_{N_{2}}\right]$, além disso, se $x=\left(x_{B}, x_{N 1}, x_{N 2}\right) \in \mathbb{R}^{n}$, sendo $x_{N 1}=0_{N 1}$ e $x_{N 2}=u_{N 2}$ tem-se que: $A x=b$ se, e somente se, $x_{B}=A_{B}^{-1}\left(b-A_{N_{1}} 0_{N 1}-A_{N_{2}} u_{N 2}\right)$.

Definição 2.1. [Solução básica de um problema primal canalizado no método Simplex] Dada uma base $A_{B}$, um vetor $x=\left(x_{B}, x_{N 1}, x_{N 2}\right)$ sendo $x_{N 1}=0_{N 1}, \quad x_{N 2}=u_{N 2} e$ $x_{B}=A_{B}^{-1}\left(b-A_{N_{1}} 0_{N 1}-A_{N_{2}} u_{N 2}\right)$ é chamado solução básica. Se $u_{B} \geq x_{B} \geq 0$ então o vetor $x=\left(x_{B}, x_{N 1}, x_{N 2}\right)$ é chamado solução básica factível.

Se $y=A_{B}^{-T} c_{B}, \quad \bar{c}=c-A^{T} y$ é o vetor de custos reduzidos. Os vetores de custos reduzidos não básicos são: $\bar{c}_{N_{1}}=c_{N_{1}}-A_{N_{1}}^{T}$ y e $\bar{c}_{N_{2}}=c_{N_{2}}-A_{N_{2}}^{T} y$.

Teorema 2.1. Se $\bar{c}_{N_{1}} \geq 0$ e e $\bar{c}_{N_{2}} \leq 0$ então uma solução básica factível, no sentido da definição 2.1, é solução ótima de $(P)$. 
Podemos resumir o papel de uma base no método Simplex considerando as condições:

$$
\text { C1 }: u_{B} \geq x_{B} \geq 0 ; \quad \text { C2 }: \bar{c}_{N_{1}} \geq 0 \text { e } \bar{c}_{N_{2}} \leq 0, \quad \text { então: }
$$

Caso 0 Se $\mathbf{C 1}$ e C2 são satisfeitas, então $(x, s)$ é solução ótima de $(P)$ e $(y, z, w)$ é solução ótima de $(D)$. Nesse caso, $A_{B}$ é base ótima tanto para o problema $(P)$ como $(D)$. Caso 1 Se $\mathbf{C} 1$ é satisfeita e $\mathbf{C 2}$ não é satisfeita, então $(x, s)$ é solução básica primal factível, nesse caso, pode-se usar o método primal Simplex para atingir a otimalidade, partindo-se de $(x, s)$. Caso 2 Se $\mathbf{C 2}$ é satisfeita e $\mathbf{C} 1$ não é satisfeita, então $(y, z, w)^{T}$ é solução dual factível, nesse caso, pode-se usar o método dual Simplex para atingir a otimalidade, partindo-se de $(y, z, w)^{T}$. Caso 3 Se C1 e C2 não são satisfeitas então $(x, s)^{T}$ não é solução factível primal e nem $(y, z, w)^{T}$ é solução factível dual.

\subsection{Base do precondicionador Separador no método de Pontos Interiores}

De acordo com as condições KKT, em cada solução ótima $\left(x^{*}, s^{*}, y^{*}, w^{*}, z^{*}\right)$ de $(1)$, se satisfaz que: $x_{i}^{*} z_{i}^{*}=0$ e $s_{i}^{*} w_{i}^{*}=0$, para cada $i=1, \ldots, n$. Diz-se que uma solução é estritamente complementar se $x^{*}+z^{*}>0$ e $s^{*}+w^{*}>0$. O teorema de Complementaridade Estrita afirma que todo problema de programação linear com solução ótima, possui pelo menos uma solução ótima estritamente complementar, veja [7].

Para cada solução de (1) são definidos os conjuntos: $\mathcal{B}\left(x^{*}, s^{*}\right)=\left\{i: x_{i}^{*}>0, s_{i}^{*}>0\right\}$ e $\mathcal{N}\left(z^{*}, w^{*}\right)=\left\{i: z_{i}^{*}>0, w_{i}^{*}>0\right\}$, e com eles, definem-se: $\mathcal{B}=\bigcup_{x^{*}, s^{*}} \mathcal{B}\left(x^{*}, s^{*}\right)$ e $\mathcal{N}=$ $\bigcup_{z^{*}, w^{*}} \mathcal{N}\left(z^{*}, w^{*}\right)$. De acordo com o Teorema de Goldman-Tucker, veja [7], os conjuntos $\mathcal{B}$ e $\mathcal{N}^{*}$ são uma partição de $\{1,2, \ldots, n\}$, isto é: $\mathcal{B} \cap \mathcal{N}=\emptyset$ e $\mathcal{B} \cup \mathcal{N}=\{1,2, \ldots, n\}$.

Em [7] é mostrado que se o problema têm infinitas soluções, a Curva Central converge ao centro analítico da face ótima e que este ponto de convergência é de fato uma solução estritamente complementar. Também prova-se que para cada solução estritamente complementar $\left(x^{*}, s^{*}, y^{*}, w^{*}, z^{*}\right)$, se satisfaz que $\mathcal{B}=\mathcal{B}\left(x^{*}, s^{*}\right)$ e $\mathcal{N}=\mathcal{N}\left(z^{*}, w^{*}\right)$. Portanto se, $\left(x^{*}, s^{*}, y^{*}, w^{*}, z^{*}\right)$ é solução do MPI primal-dual, tem-se:

$$
\mathcal{B}=\mathcal{B}\left(x^{*}, s^{*}\right) \quad \text { e } \quad \mathcal{N}=\mathcal{N}\left(z^{*}, w^{*}\right) .
$$

Antes de chegar na solução ótima usando o MPI primal-dual, pode ser natural nos perguntar se existem subconjuntos de índices semelhantes ou relacionados com $\mathcal{B}$ e $\mathcal{N}$. A resposta é afirmativa. Em [8], para cada iteração do MPI, define-se $\overline{\mathcal{B}}$ como um subconjunto de $\{1,2, \ldots n\}$ com cardinalidade $m$ tal que $A_{\overline{\mathcal{B}}}$ é não singular e $\overline{\mathcal{N}}=\{1,2, \ldots n\} \backslash \overline{\mathcal{B}}$.

De acordo com a definição acima, é claro que existe mais de uma maneira de determinar $\overline{\mathcal{B}}$, podemos nos perguntar qual é a maneira mais conveniente de escolher este conjunto de índices. Para responder essa questão, a partir de agora $(x, s, y, w, z)$ denota um ponto do MPI primal-dual e $(\hat{x}, \hat{s}, \hat{y}, \hat{w}, \hat{z})$ uma solução básica do método Simplex. A cada ponto $(x, s, y, w, z)$ de uma iteração do MPI primal-dual corresponde uma matriz $D=\left(X^{-1} Z+\right.$ $\left.S^{-1} W\right)^{-1}$ que aparece no sistema de Equações Normais que é usado para determinar uma direção de busca. Suponha que:

$$
x \rightarrow x^{*}, \quad s \rightarrow s^{*}, y \rightarrow y^{*}, \quad z \rightarrow z^{*} \text { e } w \rightarrow w^{*},
$$


onde $\left(x^{*}, s^{*}, y^{*}, w^{*}, z^{*}\right)$ é o ponto de convergência da Curva Central quando $\mu \rightarrow 0$, veja [3].

Pela continuidade das componentes da matriz $D,(3)$, (4) e o Teorema de GoldmanTucker, para cada $i \in \mathcal{B}$ tem-se que $d_{i} \rightarrow \infty$ e, para cada $i \in \mathcal{N}$ tem-se que $d_{i} \rightarrow 0$. Isto motivou a construção do precondicionador Separador. Assim espera-se que $\overline{\mathcal{B}} \approx \mathcal{B}$.

Considerando as colunas de $A$ correspondentes aos índices de $\overline{\mathcal{B}}$ e $\overline{\mathcal{N}}$, se obtém as submatrizes $A_{\overline{\mathcal{B}}}$ e $A_{\overline{\mathcal{N}}}$, respectivamente. Chamamos a matriz $A_{\overline{\mathcal{B}}}$ de Base ou matriz básica do precondicionador Separador no MPI primal-dual. A construção de $A_{\overline{\mathcal{B}}}$ não é um problema trivial, existem propostas que consideram as primeiras $m$ colunas linearmente independentes de $A$ considerando uma ordem não crescente dos elementos $D e_{j},\left\|A D e_{j}\right\|_{2}$, veja $[5,6,9]$.

Em [5], provou-se que o número de condição do sistema de Equações Normais precondicionada pelo precondicionador Separador independe da iteração do MPI primal-dual. A matriz $A_{\overline{\mathcal{B}}}$ é chamada Base de peso máximo. Em termos práticos, em [9] mostrou-se que a ordenação das colunas de $A$ considerando $\left\|A D e_{j}\right\|_{2}$ é mais eficiente no cálculo de $A_{\overline{\mathcal{B}}}$.

Uma vez obtida $A_{\overline{\mathcal{B}}}$, é considerada a permutação $\sigma=(\overline{\mathcal{B}}, \overline{\mathcal{N}}) \in \mathcal{S}_{n}$ (grupo simétrico de ordem n) com matriz de permutação associada $P: n \times n$. Reordenando as matrizes $A$ e $D$ usando $P$, tem-se: $A=\left[A_{\overline{\mathcal{B}}}, A_{\overline{\mathcal{N}}}\right] P \quad$ e $P D P^{T}=\left(\begin{array}{cc}D_{\overline{\mathcal{B}}} & 0 \\ 0 & D_{\overline{\mathcal{N}}}\end{array}\right)$, onde $D_{\overline{\mathcal{B}}}$ e $D_{\overline{\mathcal{N}}}$ são matrizes diagonais com entradas $d_{i}$ tal que $i \in \overline{\mathcal{B}}$ e $\overline{\mathcal{N}}$, respectivamente. Assim, o precondicionador Separador para o Sistema de Equações Normais é: $S=D_{\overline{\mathcal{B}}}^{-1 / 2} A_{\overline{\mathcal{B}}}^{-1}$. Observa-se que $S\left(A D A^{T}\right) S^{T}=I_{m}+W W^{T}$, onde $W=D_{\overline{\mathcal{B}}}^{-1 / 2} A_{\overline{\mathcal{B}}}^{-1} A_{\overline{\mathcal{N}}} D_{\overline{\mathcal{N}}}^{1 / 2}$. Logo, se existem suficientes índices em $i \in \overline{\mathcal{B}}$ tais que $d_{i}^{k} \rightarrow \infty$ e $i \in \hat{\mathcal{N}}$ tais que $d_{i}^{k} \rightarrow 0$, então $W$ se aproxima da matriz nula e portanto, espera-se que $S\left(A D A^{T}\right) S^{T}=I_{m}+W W^{T}$ tenha seus autovalores próximos de 1.

\section{Crossover}

Quando o MPI usa o precondicionador Separador para resolver seus sistemas lineares, surge a ideia de usar a base do mesmo para obter uma base inicial do método Simplex. O processo que calcula uma solução básica factível no sentido da Definição 2.1, a partir de um ponto do MPI, veja [8], é chamado crossover. Isto é, recuperar uma solução básica factível a partir de um ponto interior associado a uma base do precondicionador Separador.

Neste trabalho, propõe-se obter uma base inicial $B$ do método Simplex a partir de uma Base de peso máximo do precondicionador Separador, sempre que seja possível. Suponha que $(x, s, y, w, z)$ seja o ponto de uma iteração do MPI primal-dual onde foi acionado o precondicionador Separador, isto é, $A_{\overline{\mathcal{B}}}$ foi calculada.

Para cada índice $i \in \overline{\mathcal{N}}$, verifica-se se $x_{i}$ está mais próximo de 0 ou de $u_{i}$. Assim, se a comparação de distâncias: $d\left(x_{i}, 0\right) \leq d\left(x_{i}, u_{i}\right)$, define-se $\hat{x}_{i}=0$, caso contrário, define-se $\hat{x}_{i}=u_{i}$, com isto dividimos o conjunto $\overline{\mathcal{N}}$ em $\overline{\mathcal{N}}_{1}$ e $\overline{\mathcal{N}}_{2}$. Depois disso, calcula-se $\hat{x}_{\overline{\mathcal{B}}}=A_{\overline{\mathcal{B}}}^{-1}\left(b-A_{\overline{\mathcal{N}_{1}}} 0_{\overline{\mathcal{N}_{1}}}-A_{\overline{\mathcal{N}_{2}}} u_{\overline{\mathcal{N}_{2}}}\right)$, isto é, obtemos a solução básica primal $\hat{x}^{T}=\left(\hat{x}_{\overline{\mathcal{B}}}^{T}, 0_{\overline{\mathcal{N}}_{1}}^{T}, u_{\overline{\mathcal{N}}_{2}}^{T}\right), \hat{s}^{T}=\left(u_{\overline{\mathcal{B}}}^{T}-\hat{x}_{\overline{\mathcal{B}}}^{T}, u_{\overline{\mathcal{N}}_{1}}^{T}, 0_{\overline{\mathcal{N}}_{2}}^{T}\right)$. Adicionalmente, calcula-se a solução básica dual dual $\hat{y}=A_{\overline{\mathcal{B}}}^{-T} c_{\overline{\mathcal{B}}}, \quad \hat{z}^{T}=\left(0_{\overline{\mathcal{B}}}^{T}, \bar{c}_{\overline{\mathcal{N}}_{1}}^{T}, 0_{\overline{\mathcal{N}}_{2}}^{T}\right)$ e $\hat{w}^{T}=\left(0_{\overline{\mathcal{B}}}^{T}, 0_{\overline{\mathcal{N}}_{1}}^{T},-\bar{c}_{\overline{\mathcal{N}}_{2}}^{T}\right)$. Logo, são verificadas as condições $\mathbf{C} 1$ e $\mathbf{C} 2$ dadas em (2) considerando os seguintes casos: 
Caso 0 Se $\mathbf{C 1}$ e C2 são satisfeitas, então $A_{\overline{\mathcal{B}}}$ é base ótima do método Simplex primal e dual. Neste caso, o crossover não realiza nenhuma iteração do método Simplex, apenas uma omprovação de que a condição (2) é satisfeita. Caso 1 Se C1 é satisfeita e C2 não é satisfeita, então $A_{\overline{\mathcal{B}}}$ é base factível primal que será base inicial do método primal Simplex, neste caso o crossover muda do MPI primal-dual para o método primal Simplex até convergir. Caso $\mathbf{2}$ Se $\mathbf{C} 2$ é satisfeita e $\mathbf{C} 1$ não é satisfeita, então $A_{\overline{\mathcal{B}}}$ é base factível dual que será base inicial do método dual Simplex, neste caso o crossover muda do MPI primal-dual para o método dual Simplex até convergir. Caso 3 Se C1 e C2 não são satisfeitas então neste caso o crossover não é realizado, o problema é resolvido apenas usando o MPI primal-dual.

Uma questão importante no crossover é saber em que iteração do MPI primal-dual fazer a mudança para o método Simplex, usa-se o critério: $\phi(x, s, y, z, w) \leq 1$, onde

$$
\phi(x, s, y, z, w)=\frac{\left\|\left(r_{b}, r_{u}\right)\right\|}{\max (1,\|(b, u)\|)}+\frac{\left\|\left(r_{c}\right)\right\|}{\max (1,\|c\|)}+\frac{\left|c^{T} x-\left(b^{T} y-\sum_{i \in \mathcal{N}_{2}} u_{i} w_{i}\right)\right|}{\max (1,\|(b, u)\|,\|c\|)} \text {. Onde } r_{b}=b-A x,
$$

$r_{u}=u-x-s$ e $r_{c}=c+w-z-A^{T} y$. Ressaltamos que $\phi(x, s, y, z, w) \leq 10^{-8}$ é o critério de convergência do MPI.

\section{Experimentos numéricos}

Os experimentos numéricos foram realizados utilizando o software $\mathrm{PCx}$ modificado que usa métodos iterativos com uma Abordagem Híbrida (AH), veja [3], [2], e o software CPLEX. O MPI primal-dual combina o uso de dois precondicionadores: a Fatoração Controlada de Cholesky (FCC) e o Precondicionador Separador (PS). Quando o crossover é acionado, usa-se o CPLEX para terminar de resolver o problema de programação linear usando o Método Simplex (MS) seja este primal ou dual.

Tabela 1: Comparação de iterações do MS, MPI e crossover.

\begin{tabular}{|c|c|c|c|c|c|c|c|c|}
\hline \multirow{3}{*}{ Problema } & \multicolumn{5}{|c|}{ Iterações } & \multirow[b]{3}{*}{$\phi$} & \multirow{3}{*}{$\begin{array}{r}\text { MS } \\
\text { usado }\end{array}$} & \multirow{3}{*}{ Distância } \\
\hline & \multirow{2}{*}{ MS } & \multirow{2}{*}{$\begin{array}{c}\text { MPI } \\
\text { com AH }\end{array}$} & \multicolumn{3}{|c|}{ crossover } & & & \\
\hline & & & $\mathrm{FCC}$ & $\mathrm{PS}$ & MS & & & \\
\hline dano3mip & 39834 & - & 6 & 1 & 29234 & $1.95 * 10^{-2}$ & 2 & $2,4561 \cdot 10^{1}$ \\
\hline degen2 & 357 & 12 & 16 & 1 & 137 & $2.81 * 10^{-2}$ & 2 & $1,2191 \cdot 10^{-7}$ \\
\hline degen 3 & 1168 & 16 & 7 & 1 & 137 & $4.51 * 10^{-2}$ & 2 & $1,0477 \cdot 10^{0}$ \\
\hline fffff 800 & 450 & 29 & 26 & 1 & 10 & $1.20 * 10^{-6}$ & 1 & $9,7136 \cdot 10^{2}$ \\
\hline ken11 & 9828 & 22 & 20 & 1 & 1782 & $2.06 * 10^{-3}$ & 1 & $2,0045 \cdot 10^{1}$ \\
\hline kra30a & 4772548 & - & 17 & 1 & 163397 & $4.87 * 10^{-4}$ & 2 & $7,3275 \cdot 10^{2}$ \\
\hline maros-r7 & 3401 & 22 & 21 & 1 & 0 & $1.60 * 10^{-8}$ & 1 & $5,8075 \cdot 10^{5}$ \\
\hline p12345 & 401 & 15 & 11 & 1 & 1 & $1.70 * 10^{-3}$ & 2 & $1,4027 \cdot 10^{-2}$ \\
\hline p19328 & 2492 & 18 & 14 & 1 & 1 & $7.28 * 10^{-4}$ & 1 & $4,4656 \cdot 10^{-4}$ \\
\hline scr15 & 39980 & 24 & 12 & 1 & 22794 & $1.13 * 10^{-3}$ & 2 & $1,3465 \cdot 10^{0}$ \\
\hline scr20 & 98869 & 21 & 14 & 1 & 31512 & $2.84 * 10^{-4}$ & 2 & $7,4922 \cdot 10^{-1}$ \\
\hline
\end{tabular}

O problema não foi resolvido.

Na Tabela 1, apresenta-se o total de iterações do Método Simplex (MS), do Método de Pontos Interiores (MPI) e o do crossover proposto neste trabalho. Os problemas escolhidos 
são das bibliotecas MESZÁROS, NETLIB e KENNINGTON. Para determinar o início do crossover, o valor da função $\phi$ deve ser menor que 1, este valor é apresentado na sexta coluna da tabela. Na última coluna, os números 1 e 2 indicam o tipo de método Simplex que foi escolhido após uma verificação das condições $\mathbf{C 1}$ e $\mathbf{C 2}$ dadas em (2). O número 1 indica o uso do método primal Simplex e o número 2, o método dual Simplex. Na primeira e segunda colunas da Tabela 1, apresenta-se o número de iterações do MS e do MPI primaldual com AH. Já na terceira, quarta e quinta colunas apresentam-se o número de iterações realizadas no crossover. Finalmente, na coluna "Distância" exibimos a distância do ponto interior associado à base de peso máximo em relação ao vértice usado pelo Simplex.

Tabela 2: Número de iterações quando o método de escolha do CPLEX é accionada.

\begin{tabular}{|c|c|c|c|c|c|c|c|c|}
\hline \multirow{3}{*}{ Problema } & \multicolumn{5}{|c|}{ Iterações } & \multirow[b]{3}{*}{$\phi$} & \multirow{3}{*}{$\begin{array}{r}\text { MO } \\
\text { usado }\end{array}$} & \multirow{3}{*}{ Distância } \\
\hline & \multirow{2}{*}{ MS } & \multirow{2}{*}{$\begin{array}{r}\text { MPI } \\
\text { com AH }\end{array}$} & \multicolumn{3}{|c|}{ crossover } & & & \\
\hline & & & FCC & PS & $\mathrm{MS}$ & & & \\
\hline aa03 & 1996 & - & 8 & 1 & 1668 & $3.22 * 10^{-1}$ & 2 & $1,1591 \cdot 10^{-4}$ \\
\hline air06 & 1996 & - & 8 & 1 & 1668 & $3.22 * 10^{-1}$ & 2 & $1,1591 \cdot 10^{-4}$ \\
\hline cre-a & 2241 & 27 & 22 & 1 & 680 & $9.43 * 10^{-1}$ & 2 & $2,5825 \cdot 10^{3}$ \\
\hline cre-c & 1912 & 26 & 18 & 1 & 1385 & $8.27 * 10^{-1}$ & 2 & $2,3263 \cdot 10^{2}$ \\
\hline ganges & 255 & 17 & 11 & 1 & 53 & $3.21 * 10^{-4}$ & 2 & $1,5615 \cdot 10^{5}$ \\
\hline maros & 855 & 22 & 21 & 1 & 608 & $9.46 * 10^{-1}$ & 2 & $1,0434 \cdot 10^{6}$ \\
\hline model2 & 1446 & 24 & 10 & 1 & 1216 & $7.06 * 10^{-1}$ & 2 & $1,9917 \cdot 10^{2}$ \\
\hline nemsemm2 & 6282 & 42 & 35 & 1 & 1159 & $6.73 * 10^{-6}$ & 2 & $1,8616 \cdot 10^{2}$ \\
\hline nug05-3rd & 590 & - & 2 & 1 & 282 & $3.95 * 10^{-2}$ & 2 & $2,5871 \cdot 10^{-4}$ \\
\hline pcb1000 & 1548 & 25 & 10 & 1 & 534 & $5.94 * 10^{-2}$ & 2 & $2.6041 \cdot 10^{0}$ \\
\hline progas & 1039 & - & 5 & 1 & 285 & $1.20 * 10^{-1}$ & 2 & $8,417 \cdot 10^{-4}$ \\
\hline qap8 & 2210 & 10 & 3 & 1 & 2210 & $1.36 * 10^{-2}$ & 2 & $4,1587 \cdot 10^{-2}$ \\
\hline seymour & 6515 & 20 & 16 & 1 & 29 & $1.84 * 10^{-4}$ & 2 & $1,2169 \cdot 10^{1}$ \\
\hline stocfor2 & 1033 & 21 & 10 & 1 & 250 & $3.66 * 10-1$ & 2 & $5,6512 \cdot 10^{-8}$ \\
\hline $\mathrm{x} 2$ & 866 & - & 6 & 1 & 68 & $7.32 * 10^{-1}$ & 2 & $4,7831 \cdot 10^{-4}$ \\
\hline
\end{tabular}

- O problema não foi resolvido.

Na Tabela 2, permitimos que o CPLEX escolha a variante do método Simplex a ser usada. Na última coluna, o número 2 representa ao método dual Simplex. Observa-se que a partir da base obtida pelo Precondicionador Separador é possível obter uma solução factível e, portanto, o número de iterações do método Simplex é reduzido, como apresentado nas Tabelas 1 e 2. Em particular, esta redução é evidente no problema kra30a, pois são quase 5 milhões de iterações do MS comparados com 18 iterações do MPI e mais de 160 mil iterações de método Simplex dual. Destaca-se outros problemas como maros-r7, p12345 e p19328, pois o número total de iterações do MS é menor o igual a 1. Outro fato que deve-se destacar é o cálculo do PS em todos os problemas, pois só foi necessário o computo de uma base para iniciar o crossover.

\section{Conclusões}

Concluímos que usando uma base do peso máximo do Precondicionador Separador é possível achar uma solução básica para o método Simplex em grande parte dos problemas 
testados, quando isto não é possível, o problema é resolvido apenas usando o MPI primaldual. Quando o crossover é acionado o número de iterações do método Simplex é reduzido consideravelmente e ainda existem problemas que nao foram resolvidos usando apenas o MPI primal-dual, no entanto usando a abordagem do crossover isto foi possível.

Da mesma forma, acreditamos que é importante levar em consideração as diferentes modificações que devem ser feitas tanto no precondicionador FCC quanto no critério de mudança de método. Desta maneira, poderão ser desenvolvidas novas pesquisas no contexto do crossover usando a Abordagem Híbrida.

\section{Referências}

[1] L. Casacio, C. Lyra, A. R. L. Oliveira e C. O. Castro. Improving the preconditioning of linear systems from interior point methods, Computers $\&$ Operations Research. 85:129-138, 2017.

[2] M. R. Heredia, C. O. Castro e A. R. L. Oliveira. A New Hybrid Preconditioner for the Interior Point Method, TEMA, 20: 359-379, 2019. DOI: 10.5540/tema.2019.020.02.0359.

[3] M.R. Heredia e A.R.L. Oliveira, A new proposal to improve the early iterations in the interior point method. Ann Oper Res 287, 185-208, 2020. DOI; 10.1007/s10479019-03254-7

[4] F. S. Hillier e G. J. Lieberman. Introdução à Pesquisa Operacional, McGraw Hill Brasil, 2013.

[5] R. Monteiro, J. O’Neal e T. Tsuchiya, Uniform Boundedness of a Normal Matrix Used in Interior-Point Methods. SIAM Journal on Optimization, 15: 96-100, 2004. DOI: $10.1137 / \mathrm{S} 1052623403426398$

[6] A. R. L. Oliveira e D. C. Sorensen, A New Class of Preconditioners for Large-Scale Linear Systems from Interior Point Methods for Linear Programming. Linear Algebra and Its Applications, 394:1-24, 2005

[7] R. Saigal. Linear Programming: A Modern Integrated Analysis. International Series in Operations Research \& Management Science, Springer US, 2012

[8] L. Schork e J. Gondzio. Implementation of an interior point method with basis preconditioning. Mathematical Programming Computation. 2020. DOI:10.1007/s12532020-00181-8.

[9] M. I. Velazco e A. R. L. Oliveira. Computing the Splitting Preconditioner for Interior Point Method Using an Incomplete Factorization Approach.Operations Research Proceedings 2017. Springer International Publishing, pages 97-103, 2018. 